Euskal ikerketen aldizkaria | Revue d'études basques |

Revista de estudios vascos | Basque studies review

$1 \mid 1996$

Numéro I

\title{
La toponymie basque de Bayonne
}

\section{Jean-Baptiste Orpustan}

\section{OpenEdition \\ Journals}

Édition électronique

URL : http://journals.openedition.org/lapurdum/1854

DOI : 10.4000/lapurdum. 1854

ISSN : 1965-0655

\section{Éditeur}

IKER

Édition imprimée

Date de publication : 1 octobre 1996

Pagination : 9-10

ISBN : 2-84127-106-4

ISSN : $1273-3830$

\section{Référence électronique}

Jean-Baptiste Orpustan, « La toponymie basque de Bayonne », Lapurdum [En ligne], 1 | 1996, mis en ligne le 01 septembre 2010, consulté le 30 janvier 2020. URL : http://journals.openedition.org/ lapurdum/1854; DOI : 10.4000/lapurdum.1854 
Jean-Baptiste ORPUSTAN

\section{LA TOPONYMIE BASQUE DE BAYONNE}

La toponymie bayonnaise actuelle, constituée des noms des rues et des places, est presque complètement française. Il n'y aurait pas à répéter cette évidence, si un ouvrage tout récent, qui ne prétend du reste, et avec raison, à aucune compétence d'ordre linguistique pour l'étymologie, n'affirmait ceci : "Notre toponymie est gasconne, et non basque" (cf. Les rues de Bayonne. Histoire anecdotique des rues, places \& ruelles, par JeanMarie et Raymond CHABAUD, J\&D éditions, 1995, p. 9).

Cette affirmation eût été vraie s'il s'était agi par exemple du $\mathrm{XIII}^{\mathrm{e}}$ siècle : dans les noms de lieux de cette époque au Livre d'Or de Bayonne et au Livre des Établissements, soigneusement relevés dans un mémoire de DEA d'études basques soutenu à la faculté de Bayonne par $\mathrm{M}^{\mathrm{me}}$ Marinette Bidart en 1994, on compte 46 noms différents, parmi lesquels 4 ou 5 sont d'étymologie incertaine ou non romane (Ause, Doer, Bertaco, Mocoron...), les autres étant incontestablement occitans gascons, à une proportion de $90 \%$ donc. Mais depuis le XIII ${ }^{e}$ siècle, et surtout depuis que le français est devenu à son toui la langue administrative de Bayonne comme de toutes les autres vilies de France au cours des $\mathrm{XVI}^{\mathrm{e}}$-XVII' ${ }^{\mathrm{e}}$ siècles et a remplacé dans cet usage le gascon médiéval des édiles et d'une bonne partie du peuple bayonnais (qui, lui, fut sinon de tout temps, depuis longtemps en tout cas plurilingue, au moins depuis l'essor de la cité vicomtale, épiscopale et portuaire à partir du $\mathrm{XI}^{\mathrm{e}}$ siècle, car jusque-là... on ne sait pas grand-chose, et à peu près rien du point de vue linguistique), deux faits se sont produits : la ville a englobé des territoires autrefois extérieurs à elle et intégré de nouveaux noms d'une part, et très largement francisé les noms anciens de l'autre.

Le résultat est qu'aujourd'hui, sur les 484 (sauf erreur) noms relevés dans l'ouvrage cité, 84 noms seulement sont sûrement ou probablement des toponymes (parfois d'origine anthroponymique ancienne) gascons ou partiellement gascons, tandis que 16 noms environ sont des toponymes sûrement ou probablement basques. À ce stade, les très nombreux noms d'origine des célébrités et gloires locales donnés aux rues dans les derniers temps (au XIII' siècle on n'en comptait encore que trois : via de Donzag, arrue de Gahuzag, arrue de Saubainag) sont évidemment comptés comme des noms de langue française, indépendamment de l'origine linguistique (basque ou romane) des patronymes. Les proportions sont donc respectivement de $20,7 \%$ environ de noms 
autres que français, répartis en $17,4 \%$ environ de noms gascons et $3,3 \%$ de noms basques, tous ceux-ci ou peu s'en faut hors de l'enceinte de la ville médiévale. Même si la proportion des noms gascons se révèle presque six fois plus importante que celle des noms basques, on ne peut dire ni que ceux-ci sont inexistants, ni que ceux-là forment autre chose qu'une minorité restreinte à environ $1 / 6^{\circ}$ du total.

\section{Les toponymes gascons.}

L'abesque (ce toponyme gascon est cité au XIII' siècle, et s'il a été récemment "restitué", ce n'est pas "une dénomination récente" : op. cit. p. 17), Arribe-Labourt (indépendamment du nom Labourd lui-même), Arrousets, Bacheforès, Balangué, Balichon, Barrat, Barthes (ce terme local des bords des gaves est aussi entré en toponymie basque, et il n'est pas d'origine latine), Bécadine (sans doute la forme locale de "bécassine", puisque le basque qui a emprunté le nom de la "bécasse" l'appelle pecada), Beyris (comme pour les Beyrie nombreux dans la région, M. Grosclaude a montré que c'est une "vitrerie" qu'indique ce nom de provenance latine, et non une "vue", pour laquelle la toponymie romane locale, même en Cize au XIII ${ }^{e}$ siècle, n'a utilisé que le mot veder : cf. op. cit. p. 33), Biarnès, Busquet, Cabotte, Camayou (qui est sans doute un ancien "camp-mayou" ou "grand champ"), Camp-de-Prats, Cazenave, Chalibardon, (port de) Castets, Compagnet, Coumères, Couralin, Crouzade, Escanet (ce nom semble avoir une phonétique romane issue possiblement d'un nom comme *ezkandeta qui serait en toponymie basque "lieu de petits chênes", et bien que le nom Escande, comme l'a montré M. Grosclaude, soit surtout représenté en domaine languedocien de l'Aude; dans toutes nos régions landa conservé intégralement par le basque donne lane en gascon, et cette explication est non seulement linguistiquement rigoureuse, mais plus vraisemblable, vu la configuration générale de la toponymie ancienne, que l'idée de quelque "lieu où l'on égorge" ; op. cit. p. 56), Esté, Estebot, Faures (francisation de faurs comme dans beaucoup de noms bayonnais), Floride, Fraïs, Galupe, Galuperie, Gardin, Graouillats (au XIII' siècle ce nom était au singulier : arrue et port dou Graouillet), Habas (la Plaine), Hargous, Hayet, Hillans (ce nom semble plus en rapport avec le terme halha "lieu ou l'on fait le feu" et des noms régionaux comme Haillan, Halhar qu'avec "fille" peu acceptable en toponymie : op. cit. p. 70), Humère, Jacquemin (l'origine anthroponymique comme diminutif de Jacme pour "Jacques" est à peu près certaine : op. cit. p. 74), Jouandin (même type que le précédent), Lacaze, Lacoustille, Laduché (d'après M. Grosclaude la base serait le gascon lad "plateau" avec un suffixe péjoratif : op. cit. p. 80), Lagréou, Laharie, Lannot, Lartigot, Lasseguette, Lestanquet, Loung, Loustaunau (c'est évidemment "la maison neuve" et non "la petite maison" : op. cit. p. 89), Maignon, Malledaille, (pont) Mayou, Menigne-Saoube-le-Bile, Menouns, Montalibet, Mounédé (sans doute diminutif gascon de (Ar) remon), Passemillon (s'écrivait au $\mathrm{XIII}^{\mathrm{e}}$ siècle Passe melon, qui est peut-être le "petit pas (sage)" avec défor- 
mation locale de menou, et exclut de toute façon les "milans" toponymiquement très fantaisistes de R. Cuzacq : op. cit. p. 99), Mousserolles, Oelh de la Houn, Pannecau, Plachotte, Plantoun, Pontots, Pontrique, Prébendés (c'est une déformation du vieux gascon prebender), Pusterle, Lachepaillet, Sabaterie, Sablère, Sainsontan (semble constitué de l'anthoponyme, prénom et nom, Sa(i)ns Hontan), Salie, Sanguinat, Saubiole, Suseye, Talouchet, Tarrides, Téné (la forme médiévale tendes suggère le mot tender "magasinier"), Tillole, Tombeloli, Tosse, Trouillet, Tuc de Moy, Vicq.

Outre les diverses altérations de détail, plusieurs autres noms avaient au Moyen Âge des formes gasconnes francisées par la suite : arrue nave pour "Rue neuve", arrue dous Bascos (la ville portuaire et administrative du XIII ${ }^{e}$ siècle donnait aux Basques présents dans la cité, certainement depuis bien longtemps puisque la ville était encore en 1140 tellus Basclorun, "la capitale des Basques", le seul nom "ethnique" de ses noms de rue) pour "Rue des Basques", carneceirie qui doit se retrouver dans "Vieille boucherie", Port Nau pour "PortNeuf", pidloric pour "Pilori". Bien d'autres ont disparu : Berger, Estivaux, Taneiries, Noguers, Tornepique, Lague, Periceirie, Chabreire (nom occitan mais importé du périgourdin) qui sont respectivement "verger, estives, tanneries, noyers, tournepique, lagune, chaudronniers, chevrière", et quelques autres plus obscurs comme Con de baque (littéralement "corne de vache"), Mubale, Podelis (littéralement "coupe lys").

Cette toponymie bayonnaise ancienne est dominée au centre urbain par les noms de métiers et d'artisans d'une part, les noms de bateaux, de canaux et d'autres réalités liées à l'activité portuaire de l'autre. Une petite portion rappelle la présence de l'administration épiscopale, et celle des "Basques", qui ne peuvent être à cette époque que les "bascophones" reconnus comme tels, puisque le terme, "ethnique" dans ses origines, n'a alors aucune signification d'ordre administratif ou politique. $L^{\prime}$ arrue dou casted du XIII ${ }^{e}$ siècle, désignant l'actuel "château-vieux", rappelle le siège de l'ancienne "vicomté du Labourd" qui a quitté Bayonne au XII' siècle pour Ustaritz. Les toponymes extérieurs à la ville médiévale intégrés au cours de son expansion représentent des noms d'espaces et domaines ruraux, soit à base toponymique, soit à base anthroponymique ancienne, comme dans tous les pays. Ce même caractère apparaîtra dans la plupart des noms d'étymologie basque.

L'origine linguistique de quelques noms ne semble pas spécifiquement gasconne, comme le médiéval Chabreire d'origine périgourdine, ou celui de la rue Orbe, interprété habituellement par urbe "ville" avec ouverture vocalique, ou orbis "cercle", mais qui désigne certainement une rue "aveugle" c'est-à-dire "fermée", le terme étant en ce sens commun à plusieurs langues romanes dont le français (op. cit. p. 97). Ces 
noms indiquent peut-être que Bayonne a été très tôt, comme elle l'était encore au dire des voyageurs du XVIII ${ }^{e}$ siècle, un carrefour de langues. Le nom de la rue Douer est écrit au XIII ${ }^{e}$ siècle arrue de Doer : ce Doer semble donc un anthroponyme ancien (le d-initial indique en tout cas une origine indo-européenne et non basque). Bien que de phonétique apparemment gasconne, Hamboun et Limpou ne reçoivent pas d'explication satisfaisante.

Quelques autres noms laissent soupçonner quelque piste étymologique basque, en tout cas d'origine "aquitaine" non latine, assez vraisemblable :

1) Garinde (op. cit. p. 62) rappelle le village souletin de Garindein, dont la base est vraisemblablement anthroponymique : le très ancien nom de personne régional Galindo attesté des deux côtés des Pyrénées (d'Auch en Aragon) dès le IX ${ }^{c}$ siècle.

2) Ibos correspond de même au village de Bigorre, forme suffixée typique de la toponymie archaïque des mêmes régions ; mais la base est ici très probablement le mot $i b i$ que le basque a conservé au sens de "gué", présent dans d'innombrables toponymes médiévaux.

3) Bertaco cité dès le $\mathrm{XI}^{e}$ siècle (op. cit. p. 104) a une finale -co unique dans le système de toponymie bayonnaise, mais qui en basque indique un diminutif ou, plus probablement pour un lieu, un génitif "locatif" (documenté en abondance dès le haut Moyen Âge), soit "le pont de "berta", et ce "berta", on en conviendra, ressemble beaucoup au mot barthe précédemment cité, à titre d'hypothèse "pont de la barthe" ou "pont de la petite barthe".

4) Ause aujourd'hui pluralisé en "Hausses" n'a pas non plus de correspondant dans les lieux au nom roman bien identifié ; il est en revanche employé en Pays basque intérieur pour désigner des hauteurs (modestes) aussi bien que des montagnes, ce qui est de règle en toponymie : hauza, hauzo, auzku (et l'on n'est pas loin ici du nom ancien, non latin et sans doute de type basque "pré-gascon", de la ville et des habitants d'Auch, en latin Ausci), et il y a encore à rappeler haltz "aulne" qui par emploi dans la phonétique gasconne devient normalement "(h)auz". Hypothèse encore, faute de mieux.

5) Mocoron, graphie du XII' ${ }^{e}$ siècle pour l'actuel Mouqueron - et il faut en bonne méthode toujours partir des graphies les plus anciennes - n'admet pas d'explication linguistiquement acceptable ni même sémantiquement par mouquereta "petit mouchoir" (sur la même base latine mucu le basque a fait muku, mukuzu, mokanes...) et pas davantage par mousquiroun "moustique" etc. (op. cit. p. 95). En revanche le terme mokor "motte" (terre ou rocher) et le suffixe - un (puisque le gascon prononce effectivement "oun" comme dans "Baioune" !) sont parfaitement identifiés en toponymie basque médiévale, et l'hypothèse ici fortement vraisemblable donnerait "lieu de motte (s)". 


\section{La toponymie basque ancienne.}

Hors des noms incertains ci-dessus cités, les conditions de dénomination des lieux du centre urbain à partir du $\mathrm{XI}^{\mathrm{e}}$ siècle (époque où "l'évêque du Labourd" a repris le siège épiscopal longtemps abandonné) excluent la présence de noms de langue basque clairement lisibles. Ceux-ci sont donc localisés, comme beaucoup de noms romans, dans les espaces extérieurs, domaines ruraux et lieux-dits tard intégrés dans l'espace urbain. Il s'agit pour la plupart de noms toponymiques très anciens, abondamment documentés en Pays basque durant tout le Moyen Âge. D'autres sont probablement anthroponymiques, et généralement plus récents en Pays basque, et rarement antérieurs au $\mathrm{XV}^{e}$ siècle.

Il n'y a pratiquement matière à aucune hésitation pour expliquer les noms suivants :

1) Arancette (op. cit. p. 24) : romanisatior phonétique et graphique du toponyme basque médiéval arhantzeta "lieu de prunelliers".

2) Arans (idem) : même base arhantz "prunellier", mais l'absence de l'un des suffixes habituels avec ce terme laisse penser à une détérioration du nom ancien. Le mot voisin (vibrante faible non aspirée) aran "vallée" n'admet pas en général de dérivation en -s, sauf addition tardive de marque de pluriel, ce qui arrive tout de même fréquemment en toponymie basque romanisée. Absolument rien ne justifie pour autant l'expression bizarre "étymologie gasconne d'un aran qui serait "vallée" (sic! idem), car aran est, à l'indicatif obligatoire, "vallée" en basque et répandu très loin du pays bascophone actuel, désignant entre bien d'autres lieux par tautologie le "Val d'Aran", petit pays d'occitan officiel aujourd'hui, mais dont on sait bien que les habitants étaient des Basques, avant d'être romanisés, comme beaucoup d'autres Pyrénéens.

3) Aritchague (op. cit. p. 25): sauf si l'on ignore le "b.a.-ba" conjointement en toponymie, en langue basque et en langue occitane gasconne, une seule et unique étymologie est non seulement "possible" mais absolument obligatoire pour ce toponyme répété à des dizaines d'exemplaires dans tout le Pays basque médiéval, haritzaga qui est "lieu de chênes (pédonculés)". Le terme, comme le suivant Atchinetche et d'autres, a subi la palatalisation (chuintement) de la sifflante d'origine, seule altération de la phonétique locale, mais qui procède, comme on le verra... du basque. Un autre problème est de savoir si ce terme a à voir avec le toponyme médiéval Urruzaga forme régulièrement assimilée du plus commun urritzaga (voir plus loin) qui apparaît dès le $\mathrm{XII}^{c}$ siècle au Livre d'Or, et en ce cas, qui reste à prouver, il y aurait eu substitution. Mais les deux noms étaient cités dans les environs de Bayonne au Moyen Âge.

4) Atchinetche (op. cit. p. 27) : les erreurs d'analyse sont tout à fait incompréhensibles pour ce classique de la toponymie (ou "domonymie") basque médiévale d'une extrême simplicité : aitzintetxe "maison située à l'avant". Et bien entendu il n'y a aucune "racine atchi" (idem) 
dans Latxague (tous les noms "La(t)xague" sont des romanisations phonétiques des maisons médiévales Latsaga, dont l'une des plus fameuses était celle d'Asme en Ostibarret ; son seigneur "Pès de Laxague" familier de la cour de Charles III de Navarre à la fin du XIV ${ }^{e}$ siècle et aussi... marchand bayonnais !), Latche, Lasse, Laxia et autres noms locaux de ce type, mais bien la base toponymique basque lats "cours d'eau", elle aussi reproduite à des centaines et des centaines d'exemplaires dans toute la région, et fort loin du territoire bascophone actuel comme dans la toponymie basque médiévale elle-même. La traduction gasconne de ce nom, tout aussi répandue, est Casedevant.

5) Bouroua (op. cit. p. 34) : la méconnaissance totale du basque et les préjugés anti-basques de R. Cuzacq - l'auteur de ces lignes l'a eu comme professeur d'histoire au lycée de Bayonne - étaient fort connus, mais ils n'autorisent en rien les fantaisies linguistiques qui feraient venir ce nom de quelque mot gascon; en tout cas bourrouilh est doublement exclu : sémantiquement (on n'a jamais connu un lieu nommé par le "désordre" !) et phonétiquement (double -rr- au lieu du simple, disparition de -ilh- final, adjonction mystérieuse d'un -a final... c'est beaucoup d'obstacles à la fois). Sous cette graphie francisée, c'est simplement burua "la tête" et en toponymie "l'extrémité, la limite"; le mot est rarement employé seul, mais l'exemple de la maison médiévale de Mixe Buruco qui a laissé des noms d'état civil ne permet pas d'explication plus acceptable.

6) Chala (op. cit. p. 40) : issu d'un mot germanique, le terme qui a donné le basque sala est commun à toute la région, et avait à l'origine le sens de "maison noble", assez vite dilué pour désigner des annexes ou des fondations de ces mêmes maisons; le curieux est la "chuintante" initiale qui rend dans la phonétique romane bayonnaise et péribayonnaise la sifflante basque "apico-alvéolaire" du mot, et dont il sera traité plus loin vu son extension.

7) Char (op. cit. p. 42) : cette ancienne ferme a dû porter le nom basque sarri extrêmement répandu en toponymie médiévale pour désigner des lieux de végétation dense (plutôt que sara "taillis, bosquet" qui a fait le nom de Sare entre bien d'autres), et qui a laissé aussi le nom béarnais de l'ancien Oloronais (où la toponymie basque est importante) Charre avec la même chuintante initiale que Charritte (basque Sarrikota) etc.

8) Chauron (op. cit. p. 43) : si le mot vient, comme l'affirme l'ouvrage cité, du "gascon chourrou qui désigne l'eau qui sourd de terre", deux remarques s'imposent : $1^{\circ}$ c'est là un mot de type expressif qui n'a par luimême, comme tous les mots de ce genre, aucune appartenance étymologique précise (cf. le français susurrer, le castillan churro, le basque xurruxta etc.); $2^{\circ}$ mais c'est aussi un mot très banal du lexique basque pour désigner "l'évier" xurruta, et, ce qui est plus intéressant, un mot nommant des maisons médiévales probablement situées près d'un écoulement d'eau : deux maisons en Ostibarret au XIV siècle dont une noble citée en 1304, et bien d'autres maisons citées plus tard. Mais la forme Chauron 
suppose beaucoup d'altérations phonétiques et graphiques de ce terme initial, et il vaut mieux songer à une autre origine.

9) Chouhour (idem): ce nom exclut évidemment toute altération du précédent xurrut, et reproduit exactement, avec la même "chuintante" locale, le mot basque zuhur "sage", et c'est très probablement comme pour beaucoup d'autres noms de la région un "surnom" (le "sage", mais aussi par approximation sémantique "parcimonieux" puis "avare", sens sous lequel il est encore ordinairement employé), celui d'un propriétaire ou habitant de ce domaine, selon un procédé d'appellation très répandu tant pour les noms basques que romans.

10) Duret : (op. cit. p. 53) : le nom de cette "ferme", s'il était d'origine locale, était très probablement un Ureta "lieu d'eau" (peut-être urreta "lieu de coudriers", le mot urr étant présent aussi au Livre d'Or pour nommer le témoin Urruzaga en 1125, forme plus courante "urritzaga"), qui a subi deux petites altérations romanes extrêmement banales : adjonction de la préposition d'origine en tête, et effacement de la voyelle atone (en gascon) finale ; comme le voisin Anglet, Viscarret en Navarre, et dans les noms d'état civil Ouret, Iratchet etc., tous issus de dérivés basques locatifs en -eta.

11) G(u)ibéléou (op. cit. p. 64) : si la syllabe finale indique peut-être une adaptation (plutôt qu'une "étymologie" qui exigerait une analyse plus rigoureuse) au gascon local, la graphie de Cassini suggère que la base est gibel "arrière", terme (et peut-être lieu ?) opposé à aitzin précédemment cité ; il est présent en toponymie médiévale basque.

12) Haur(r)ena (op. cit. p. 69) : limpide dérivation basque, soit à valeur possessive "celui (c'est-à-dire le domaine, la maison) de l'enfant", étant donné l'emploi de haur "enfant" pour former d'innombrables prénoms-surnoms dans les familles basques, soit à valeur superlative à base aurr "antérieur, situé avant" (d'où dans nos dialectes aurki "avers"), terme qu'on trouve dans les maisons médiévales de Baïgorry, et au sens de "le plus en avant".

13) Ilbarritz (op. cit. p. 72) : sous cette forme connue aussi sur la côte biarrote le nom est peu explicite, mais c'est à coup sûr un composé basque ancien.

14) Lahubiague (op. cit. p. 81) : la plupart des finales -ague des noms de lieux et d'état civil régionaux, peut-être tous, sont issus de la romanisation du suffixe locatif basque archaïque -aga (la langue ne l'utilise plus au moins depuis le Moyen Âge et il est aujourd'hui fossilisé à des centaines d'exemplaires en noms de lieux et d'état civil). Ici la base est soit le banal toponyme basque hobiaga "lieu de fosses", soit le tout aussi répandu lohiaga "lieu limoneux, boueux", que le Livre d'Or cite justement au $\mathrm{XIII}^{c}$ siècle sous la forme régulière en orthographe latinisante du temps ("f" pour " $h$ ") de Lofiague. "Lahubiague" a un proche parent dans le luzien Lohobiague, dont les voyelles n'ont pas subi d'altération romane, mais une "haplologie" ou chute de l'une de deux syllabes d'articulation identique ou proche (c'est un procédé extrê- 
mement répandu en phonétique basque, ancienne et moderne) : ce nom est en effet issu à coup sûr d'une composition primitive lohihobiaga, c'est à dire "lieu des fosses boueuses", ce qui, on en conviendra, va aussi bien à Lohitzun, d'où dérive le "Luz" roman, qu'au sol des marais bayonnais !

15) Raspure (op. cit. p. 108) : encore un nom parfaitement transparent sous l'aphérèse romane qui lui a supprimé la voyelle initiale et l'affaiblissement de la voyelle atone finale (Phagabure, Ibarboure, Mogabure etc.), issu d'un iratzpuru "limite de la fougeraie" ; on ne peut exclure qu'un ancien haritzpuru "limite des chênes" ait pu subir une métathèse en langue romane (car les Basques, et pour cause, ne peuvent confondre haritz et iratz!), mais c'est peu probable.

16) Sabalce (op. cit. p. 112) : vu le lieu désigné, l'incertain "propriétaire" trouvé par R. Cuzacq "dans les environs" devait, à une époque certainement ancienne mais non précisée, porter selon l'usage le nom de son domaine, "lieu plat", sens du toponyme médiéval Zabalza, l'un des noms anciens de Saint-Jean-le-Vieux et nom de plusieurs maisons médiévales, et qui caractérise aussi cette zone de plaine.

Les toponymes basques, très bien caractérisés et parfaitement reconnaissables pour la plupart, enserrent la ville médiévale dans tout l'espace qui regarde vers le Labourd intérieur. Comme ils sont très anciens, presque tous déformés peu ou prou par l'usage en langue gasconne urbaine, ils donnent la preuve que la ville fut bâtie à une époque où la zone était encore bascophone, ce que les noms anciens de la ville elle-même, Lapurdum, Lapurdi, Laburdi, Laburdensis... et Baiona, Baione, Bayonne, qui ne sont pas des mots explicables par le latin (pas plus que Aturris, en basque Aturri) quoique morphologiquement latinisés selon les habitudes des Romains, à plus forte raison romans, laissent entendre assez clairement.

\section{Les altérations et palatalisations phonétiques du gascen bayonnais.}

Il y aurait peut-être dans la toponymie bayonnaise trace d'un fait phonétique au demeurant extrêmement répandu, mais qui a une extension particulière en basque, et se lit parfois en toponymie médiévale : c'est le passage de la consonne nasale $n$ à la latérale $l$, cette "dénasalation et latéralisation" (parfois l'inverse ou "nasalisation") facilitée par l'articulation apicale des deux phonèmes s'illustre par le mot français "poterne", alors que le gascon maintient la latérale originelle du latin posterula dans l'ancien posterle (1266) aujourd'hui pusterle. Mais le nom Mosseirole actuellement Mousserolles pourrait s'expliquer par une altération de ce type pour le mot occitan nommant le "mousseron", et de même le melon de 1266 à partir de menon. Ces faits sont à confirmer; alors que la toponymie basque offre clairement, dans des textes romans écrits tant en navarro-castillan qu'en gascon, les faits suivants, où l'altération ne se fait pas seulement à l'initiale de mot que signale 
L. Michelena dans sa Fonética Histórica Vasca (p. 324 et 550-552) : pour le mot aintzin "avant" dans elcinecoeche (1350), pour le mot itzain "bouvier" (ou l'inverse pour itzal "ombre" ?) dans yzaleche (1347) à côté de $y$ dçaneche (1350 : c'est la même maison à Jaxu), pour le mot intzaur "noix" dont les altérations suivantes sont les unes labourdines et les autres bas-navarraises dans ilçaurzpe (1350 : fréquent à cette date), alsaurspy (1505 à Sare), elsospea (1505 à Saint-Pée), le mot eltzaur étant même entré dans le lexique usuel sous diverses formes dialectales à côté du commun intzaur.

Ce qui est infiniment plus typique de l'ancienne toponymie bayonnaise et environnante, c'est la "palatalisation" ou "chuintement" des sifflantes dans les noms basques, étendue à tout le domaine roman péribasque, comme le montrent les exemples de Charre et Charritte déjà cités. Il semble que ce procédé ait été quasi systématique dans le traitement que le gascon bayonnais a fait des toponymes basques: en témoignent Atchinetche, Aritchague, Chala, Char, Chouhour. Le basque utilisait dès le Moyen Âge la palatalisation expressive (qui pour les sifflantes donne les chuintantes écrites en français $\mathrm{ch} / \mathrm{tch}$ mais touche bien d'autres phonèmes en basque) pour donner aux mots une valeur "hypocoristique", affective, diminutive etc., et elle s'est encore considérablement étendue (indépendamment des palatalisations résultant des simples changements phonétiques propres à certaines zones dialectales) dans la langue moderne.

Parfois, les mots employés en gascon montrent que le ch est la réalisation de l'apico-alvéolaire basque de sarri (qui est la sifflante sourde correspondant à la sonore du français je), comme dans Char, Charre, Charritte précédemment cités. Mais c'est la sifflante dorso-alvéolaire, celle du français sou qui est palatalisée dans Atchinetche, Aritchague, Chouhour, correspondant à aitzin, haritz, zuhur. Cette tendance phonétique assurément issue de l'influence de la langue basque sur le gascon parlé à Bayonne et autour, qui a même laissé des vestiges dans le parler local actuel, s'illustre de la façon la plus éclatante dans un nom d'origine purement gasconne comme la Plachotte ou "petite place" avec le suffixe diminutif-ote, mais qui a reçu un second diminutif expressif avec le chuintement de la sifflante : le nom moderne témoigne ainsi de trois langues, le gascon du mot et surtout de son suffixe, le basque de la chuintante elle aussi "diminutive", et le français de l'orthographe à double -tt(e). Ce pourrait être un exemple typique du multilinguisme ancien de la population bayonnaise ; mais le fait s'étend bien au-delà jusqu'à Biarritz, dans les lieux où le parler roman est venu se mélanger à l'ancienne langue, et parfois, au moins dans les zones les plus urbanisées, la supplanter dans certaines couches de la population dès la fin du Moyen Âge.

\section{Noms d'origine récents, basques et autres.}

Les 484 noms de rues et places de Bayonne sont constitués en majorité de noms de personnages illustres ou notables issus de la 
région, portant des noms parfois d'origine gasconne, parfois d'origine basque et aussi bien d'autres. La présente note ne serait pas complète si, à côté des vrais toponymes anciens, il n'était pas fait mention aussi des noms de personnes d'origine basque, qui sont de vrais toponymes basques, toujours issus comme on le sait des noms de maisons, et de maisons qui en général existent encore dans le pays de langue basque, qui commençait naguère aux portes mêmes de la ville comme on vient de le voir.

Il n'importe en rien que ces personnages eux-mêmes aient été ou non bascophones, et qu'inversement des personnages au nom roman aient été des "Basques bascophones", comme cela arrive aujourd'hui comme autrefois. Mais du moment que leur nom témoigne de leurs origines basques, eussent-ils vécu toute leur vie fort loin du pays, on peut leur appliquer sans risque d'erreur le vers de la fable : "Si ce n'est toi, c'est donc ion père..." ou... ton grand-père, et même si tel parmi eux (op. cit. p. 49) fait partie de l'Académie gascoune! On pourra chercher quelles catégories, parmi ces célébrités locales, chanoines, maires, médecins, militaires, etc. portent le plus grand nombre de noms basques, ou gascons, ou espagnols, ou autres, et dessiner ainsi une sorte de socio-géographie anthroponomastique bayonnaise.

Les noms basques d'origine sont, sauf erreur, au nombre de 21 :

Jauréguiberry (amiral), Sala (amiral : son père était notaire à Ossès, son lieu d'origine, op. cit. p. 22), Harambillet (André), Oyhénart (Arnaud d'), Chaho (Augustin), Lacarre (évêque), Tambourindéguy (Bernard), Oyarzun (Carlito), Borthayre (chanoine), Daranatz (chanoine), Subigaraychipi (nom du corsaire "Croisic"), Etcheverry (Denis), Etcheverlepo (André ; le nom désigne la maison située au "col d'Etcheberri", et la forme "Etcheverlopo" citée est une altération : op. cit. p. 56), Arbeletche (Henri), Hiriart (maire), Basterrèche (maire de l'époque révolutionnaire), Latxague (Joseph), Lafaurie-Dechepare (seul le deuxième patronyme est basque évidemment, le premier gascon), Harispe (maréchal), Narbaïts (Sauveur), Iribarne (sous-lieutenant).

Il est piquant de noter que la "jeune fille qui sauva la ville" en 1638 , selon un légendaire urbain qui va de Paris (Geneviève) à Nice (Ségurane) et autres lieux, portait le nom patronymique très basque de Garay (op. cit. p. 92). Enfin la langue basque est encore représentée dans la toponymie moderne de Bayonne, mieux que par le lieu commun Toki-Eder qui traduit le non moins banal "Beaulieu" (op. cit. p. 118), par les noms de toutes les montagnes qui se dessinent à l'horizon: Ahusqui, Arsamendi, Baïgura (qui n'est pas du tout à "Saint-Etienne-deBaïgorry", mais sépare la vallée d'Ossès et le pays d'Irissarry de Hélette et des villages labourdins Mendionde, Macaye et Louhossoa : op. cit. p. 28), Iparla, Mondarrain, La Rhune (qu'il faudrait écrire en un seul mot Larhune et non "la Rhune" comme on persiste à l'écrire, car il ne s'agit en rien de l'article français : op. cit. p. 110), Ursuya.

Plusieurs noms, anthroponymes pour la plupart, représentent aussi l'hispanité bayonnaise, dans laquelle comptent pour beaucoup les réfu- 
giés des grands drames politiques péninsulaires à commencer par l'expulsion des Juifs : Gomez, Diesse (op. cit. p. 49), Froix, Furtado, Feria, Pé-de-Navarre.

En somme la toponymie bayonnaise actuelle figure assez bien, autour d'un noyau réduit mais encore bien dessiné hérité de la ville médiévale et de son administration de langue romane, l'histoire humaine et linguistique, complexe et disparate comme dans toute ville à la fois portuaire et frontalière.

N.B. - Pour l'onomastique romane gasconne, il faut se reporter aux deux ouvrages fondamentaux de Michel Grosclaude : Dictionnaire étymologique des noms de famille gascons, Pau 1992, et Dictionnaire toponymique des communes du Béarn, Pau 1991.

Jean-Baptiste ORPUSTAN

Université Michel de Montaigne - Bordeaux III ERS 142 du CNRS 


\section{Laburpena}

Baionako leku-izenak, karrika, zubi eta plaza, XIII-garren mendean gaskoinez ziren gehienak, 46 ezagunetarik lauzpabortz salbu. Baina, geroztik frantseserat itzuliak izan dira, eta frantsesetik eta frantsesez eman izen berriak, ezpaitaiteke erran beraz, berriki agertu den liburu batek diona (Les rues de Bayonne...) : Baionako leku-izendegia gaskoina dela oraino. Xehekiago, liburu hortan aipatzen eta aztertzen diren 484 izenetarik $\% 80$ frantsesez dira, gaineratekoetan $\% 17,4$ gaskoinez geldituak, eta ere \% 3,3 euskaldunak, orotara 84 izen gaskoin guti edo aski aldaturik, eta 16 euskal izen, gehienean azter errexak, erdararen eraginez ukan dituzten itxuraldatzen azpian.

Euskaldunak Baionan baziren betidanik (Kompostelako beilariak Euskaldunen hiri nagusitzat dauka XII-garren mende hastapenean, XIII-garrenean orai bezala badu jadanik edo... oraino "Euskaldunen karrika"...), hiri barneko izen zaharretan bizpahiru dira erdalkira ezin bilduak. Erdi-Aroko hiri ttipiaren harresi inguruetan hasten ziren berehala euskal izenak : laboretxe, leihor, mendixka, ur-bazter eta lohi ; geroztik hiria hedatu denean barneratu baititu. Egiazko leku-izen zahar horien gainera, izendegi berriak sortu ditu izenak euskaratik, frantsesez eman badira ere : alde batetik hiriko edo erresumako jaun aipatu batzuen izenak, hogei bat; eta horietan andererik ezpada edo guti, ohartzeko da gaskoinez "hiria salbatu zuen nexkañoaren" izena, 1638an omen, Garay zela... ; bertzetik Baionako leku-izenek erakusten duten gaskoin ahoskerak badu zerbait euskararenetik biziki hurbil dena : konsonant zenbaiten, "ttipitasun" edo "amultsutasunez", sabaikatzea : Aitxinetxe, La Plachotte.

Jean-Baptiste ORPUSTAN

Université Michel de Montaigne - Bordeaux III ERS 142 du CNRS 